\title{
Spiral Hierarchical Superstructures from Twisted Ribbons of Self-Assembled Chiral Block Copolymers
}

\author{
By Kai-Chieh Yang and Rong-Ming Ho* \\ rhmo@mx.nthu.edu.tw \\ $\dagger$ Department of Chemical Engineering, National Tsing Hua University, Hsinchu 30013, \\ Taiwan
}

\section{$\underline{\text { Supporting information }}$}

\section{Materials preparation}

Figure S1 Synthetic routes of PS-PLLA using two-step polymerization starting with atom transfer radical polymerization of PS followed by ring-opening polymerization of PLLA.

Figure S2 Comparison of GPC curves (for sample of Table S1 entry 1) between (a) PS-OH and (b) PS10-PLLA24 synthesized through ring opening polymerization using (a) as a macroinitiator.

Figure S3 Comparison of GPC curves (for sample of Table S1 entry 2) between (a) PS-OH and (b) PS17-PLLA42 synthesized through ring opening polymerization using (a) as a macroinitiator.

Figure S4 Comparison of GPC curves (for sample of Table S1 entry 3) between (a) PS-OH and (b) PS24-PLLA67 synthesized through ring opening polymerization using (a) as a macroinitiator.

Figure S5 $\quad{ }^{1} \mathrm{H}$ NMR spectrum of PS10-PLLA24. The measurement was carried out using deuterated chloroform as a solvent at $25^{\circ} \mathrm{C}$.

Figure S6 $\quad{ }^{1} \mathrm{H}$ NMR spectrum of PS17-PLLA42. The measurement was carried out using deuterated chloroform as a solvent at $25^{\circ} \mathrm{C}$.

Figure S7 $\quad{ }^{1} \mathrm{H}$ NMR spectrum of PS24-PLLA67. The measurement was carried out using deuterated chloroform as a solvent at $25{ }^{\circ} \mathrm{C}$.

Table S1 Characterization of synthesized PLLA-rich PS-PLLA samples with various molecular weights.

\section{Thermal Properties}


Figure S8 TGA thermograms of (a) PS10-PLLA24, (b) PS17-PLLA42 and (c) PS24PLLA67. The heating rates are $10{ }^{\circ} \mathrm{C} \mathrm{min}^{-1}$.

Figure S9 DSC thermograms of (a) PS10-PLLA24, (b) PS17-PLLA42 and (c) PS24PLLA67 samples. The heating rates are $10^{\circ} \mathrm{C} \mathrm{min}^{-1}$.

\section{Low magnification TEM image for curved multilayered lamellae}

Figure S10. Low magnification TEM micrograph of self-assembled PS24-PLLA67 from solution casting with solvent evaporation rate of $0.04 \mathrm{ml} \mathrm{hr}^{-1}$ giving curved multilayered lamellae wiht both concentric and roll-cake texture.

\section{Examination of crystallization effects on self-assembled hierarchical superstructures}

Figure S11 1D WAXD profiles of solution-cast PS24-PLLA67 $\left(f_{\mathrm{PLLA}^{\mathrm{v}}}=0.70\right)$ after thermal annealing at $180{ }^{\circ} \mathrm{C}$ followed by rapid cooling at $150{ }^{\circ} \mathrm{C} \mathrm{min}{ }^{-1}$ to 0 ${ }^{\circ} \mathrm{C}$. The solution-cast samples were prepared by solution casting with solvent evaporation rate of (a) $0.4 \mathrm{ml} \mathrm{hr}^{-1}$; (b) $0.04 \mathrm{ml} \mathrm{hr}^{-1}$; (c) $27 \mathrm{mml} \mathrm{hr}^{-1}$.

\section{Templated sol-gel reaction of multilayered lamellar structure}

Figure S12 SEM micrographs of nanostructured $\mathrm{SiO}_{2}$ fabricated by templated sol-gel reaction using nanoporous PS as a template prepared by hydrolysis of selfassembled PS24-PLLA67 with concentric lamellar texture (a) and roll-cake texture (b), followed by removal of PS through UV exposure. The insets show the corresponding illustrations of the forming textures.

\section{Morphological evolution of PLLA-rich PS-PLLA from self-assembly}

Figure S13. (a)TEM micrograph and (b) SAXS result of self-assembled PS24-PLLA67 from solution casting with extremely slow solvent evaporation rate at $0.0008 \mathrm{ml} \mathrm{hr}^{-1}$. Undulated lamellae with long-range order can be observed by TEM along with the appearance of reflections at high $q$ region at which the $d$-spacing was approximately $54 \mathrm{~nm}$ determined by the primary reflection in the SAXS profile. The inset shows the corresponding illustration of the forming texture.

7. PLLA-rich PS-PLLA samples with equivalent volume fraction but different molecular weights

Figure S14 TEM micrographs of self-assembled (a) PS10-PLLA24 and (b) PS17PLLA42 from solution casting after thermal annealing at $180{ }^{\circ} \mathrm{C}$ followed by rapid cooling at $150{ }^{\circ} \mathrm{C} \mathrm{min}^{-1}$ to $0{ }^{\circ} \mathrm{C}$. The PS microdomains appear dark 
and PLLA microdomains appear bright due to $\mathrm{RuO} 4$ staining.

Figure S15 1D SAXS profiles of self-assembled PS24-PLLA67, PS10-PLLA24 and PS17-PLLA42 from solution casting after the thermal treatment with the same sample preparation procedure.

8. Evidence for inter-chain chiral interactions of self-assembled PLLA-rich PS-PLLA

Figure S16 VCD and corresponding FTIR absorption spectra of C-O-C vibrational mode of PLLA-rich PS24-PLLA67 in the (a) DCM solution and (b) solid states. The concentration of solution is $2 \mathrm{wt} \%$.

\section{Anisotropic effect on VCD spectrum}

Figure S17 VLD and corresponding FTIR absorption spectra of C-O-C vibrational mode of PLLA-rich PS24-PLLA67 in the (a) DCM solution and (b) solid states. The concentration of solution is $2 \mathrm{wt} \%$. 


\section{Material preparation}

As illustrated in Figure S1, PLLA-rich PS-PLLA samples were prepared by a doubleheaded polymerization for two-step polymerization, atom transfer radical (ATRP) and ringopening polymerization (ROP) in sequence. The synthesis of PS-PLLA with PLLA as a major composition is challenging due to the difficulty for dissolving crystallizable PLLA in the common solvent for PS, resulting in the precipitation of the product during polymerization of the PLLA block. Moreover, the probability of the PS with hydroxyl chain end severing as a macroinitiator for the polymerization of the PLLA will decrease as molecular weight increases, resulting in the low propagation of PLLA polymerization that might cause the occurrence of PLLA homo-polymerization. To solve the problems, a synthetic route with low monomer conversion of L-lactide as low as 0.5 was carried out; by combination of fractionation using acetonitrile as a solvent to remove unexpected products such as PLLA homopolymer, it is feasible to synthesize the PS-PLLA with controlled constituted fraction and narrow polydispersity for self-assembly.

The BCPs* were synthesized using PS end-capped with hydroxyl group, PS-OH, as macroinitiator and $\mathrm{Sn}(\mathrm{Oct})_{2}$ as a catalyst. For the synthesis of PLLA-rich PS-PLLA samples, $0.1 \mathrm{~g}$ PS-OH, $0.35 \mathrm{~g}$ L-lactide, $8 \mathrm{mg} \mathrm{Sn}(\mathrm{Oct})_{2}$, and $1.5 \mathrm{ml}$ dry toluene were added in the dry glass flask equipped with a magnetic stirrer in glovebox. The flask was set in an oil bath at 110 ${ }^{\circ} \mathrm{C}$ stirring for 5 hours, and then cooled down to the room temperature rapidly through ice bath. The obtained polymers were then dissolved in $\mathrm{CH}_{2} \mathrm{Cl}_{2}$ followed by precipitation in methanol. The synthesized PS-PLLA samples were then purified by fractionation using acetonitrile as solvent to remove unexpected products such as PLLA homopolymer. The successful syntheses of the BCPs*, including PS10-PLLA24, PS17-PLLA42, and PS24-PLLA67, via chain extension of PS-OH with lactides were demonstrated in comparison with the respective GPC curves (Figure S2-S4). Figure S5-S7 display the ${ }^{1} \mathrm{H}$ NMR spectra of the PS10-PLLA24 $\left(\mathrm{M}_{\mathrm{n}}\right.$ $=34,100 \mathrm{~g} / \mathrm{mole}, \mathrm{Ð}=1.28)$, PS17-PLLA42 $\left(\mathrm{M}_{\mathrm{n}}=58,700 \mathrm{~g} / \mathrm{mole}, \mathrm{Ð}=1.31\right)$, and PS24PLLA67 $\left(\mathrm{M}_{\mathrm{n}}=91,000 \mathrm{~g} / \mathrm{mole}, \mathrm{Ð}=1.30\right)$, respectively. ${ }^{1} \mathrm{H}$ NMR spectrum of PS-PLLA assignments are as follows: ${ }^{1} \mathrm{H}$ NMR $\left(500 \mathrm{MHz}, \mathrm{CDCl}_{3}\right): \delta=6.46-7.20(5 \mathrm{H}, \mathrm{ArH}), 5.12-5.16$ (q, $1 \mathrm{H}, \mathrm{CH}\left(\mathrm{CH}_{3}\right)$ ), 1.82 (backbone, $\left.{ }^{1} \mathrm{H}, \mathrm{CH}\right), 1.58$ (d, 3H, $\mathrm{CH}\left(\mathrm{CH}_{3}\right)$ ), 1.40 (backbone, $2 \mathrm{H}, \mathrm{CH}_{2}$ ). Accordingly, the relative volume fractions of PS-PLLAs synthesized were determined by comparing the relative intensity of the respective ${ }^{1} \mathrm{H}$ resonances at which the densities of PS and PLLA are 1.05 and $1.25 \mathrm{~g} \mathrm{~cm}^{-3}$. The data determined were summarized in Table S1. As a result, PLLA-rich PS-PLLAs with equivalent volume fraction but different molecular weights as listed in Table $\mathbf{S 1}$ were synthesized.

For the examination of self-assembled morphologies, solution casting of the PS-PLLA was carried out at room temperature using dichloromethane as a solvent. Bulk samples were prepared by solution casting from dichloromethane $(10 \mathrm{wt} \%)$ at room temperature. Samples 
were first dissolved in dichloromethane and filtrated through a syringe filter with $0.45 \mu \mathrm{m}$ pore size to remove the impurities. The solution was then transferred in a vial and sealed with aluminum foil. Subsequently, several holes were punched on the aluminum foil using needle for the evaporation of the solvent at which the solvent evaporation rate can be controlled by the numbers of the holes created. After casting, the solution-cast samples were thermally treated at $180{ }^{\circ} \mathrm{C}$ for $5 \mathrm{~min}$ to annihilate the crystalline PLLA followed by rapid cooling at 150 ${ }^{\circ} \mathrm{C} \min ^{-1}$ to $0{ }^{\circ} \mathrm{C}$. As evidenced by wide-angle X-ray diffraction (WAXD) results (Figure S8), no significant crystalline diffraction of the PLLA can be identified, suggesting no PLLA crystallization effects on self-assembled results from microphase separation after the thermal treatment.
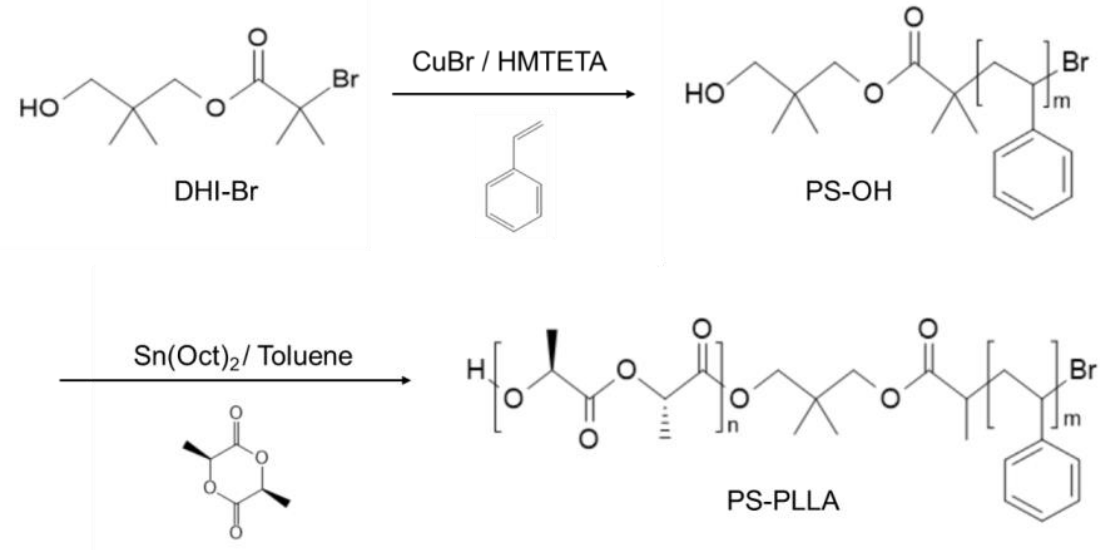

Figure S1. Synthetic routes of PLLA-rich PS-PLLA using two-step polymerization starting with ATRP of PS-OH followed by ROP of PLLA.

(b)

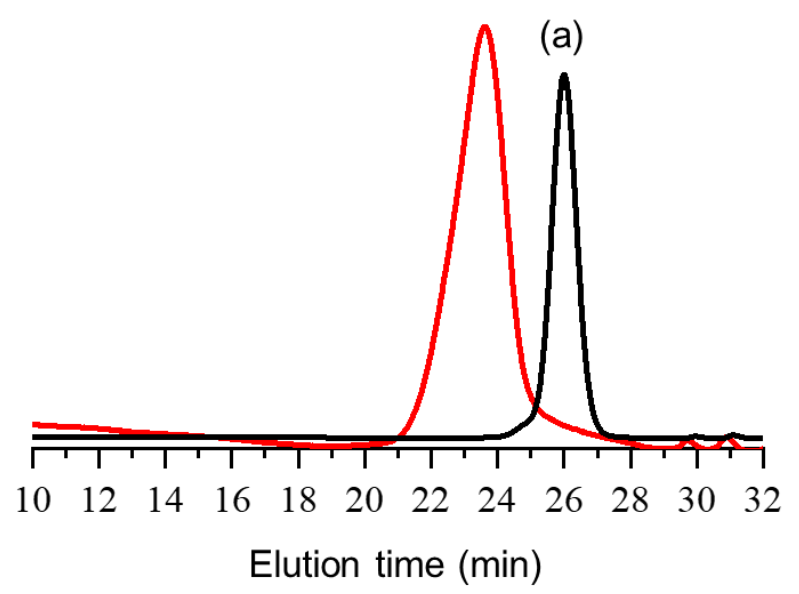

Figure S2. Comparison of GPC curves (for sample of Table S1 entry 1) between (a) PS-OH and (b) PS10-PLLA24 synthesized through ring opening polymerization using (a) as a macroinitiator. 


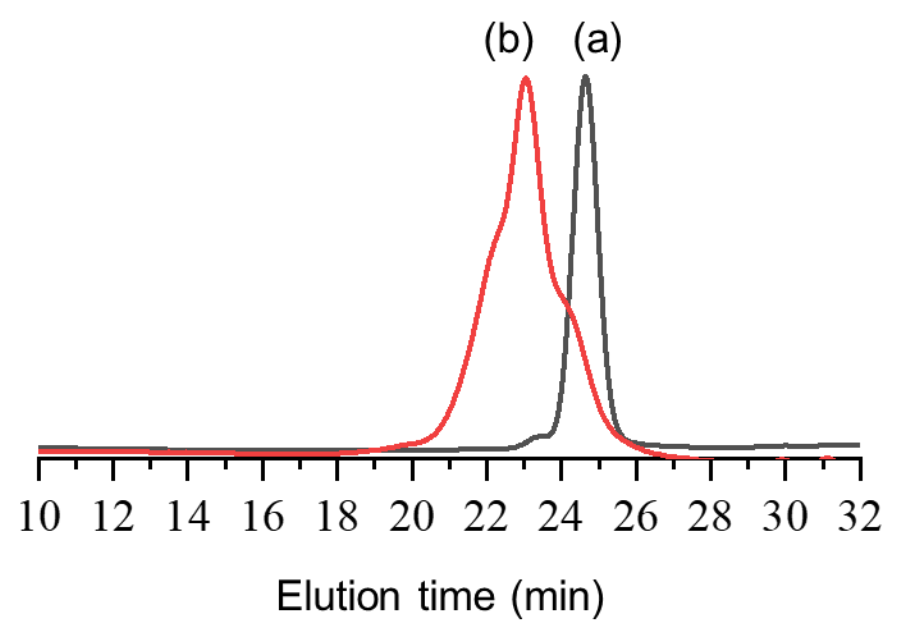

Figure S3. Comparison of GPC curves (for sample of Table S1 entry 2) between (a) PS-OH and (b) PS17-PLLA42 synthesized through ring opening polymerization using (a) as a macroinitiator.

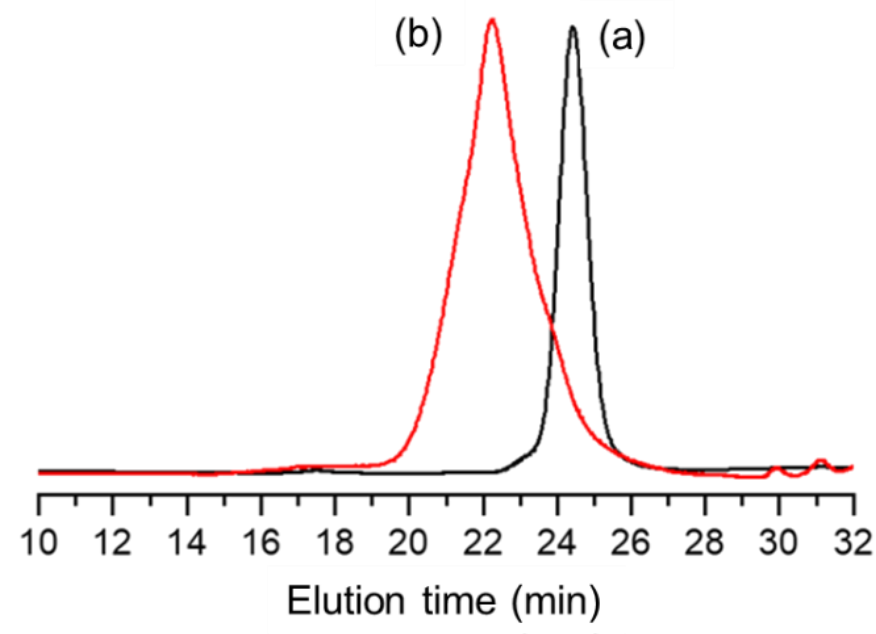

Figure S4. Comparison of GPC curves (for sample of Table S1 entry 3) between (a) PS-OH and (b) PS24-PLLA67 synthesized through ring opening polymerization using (a) as a macroinitiator. 


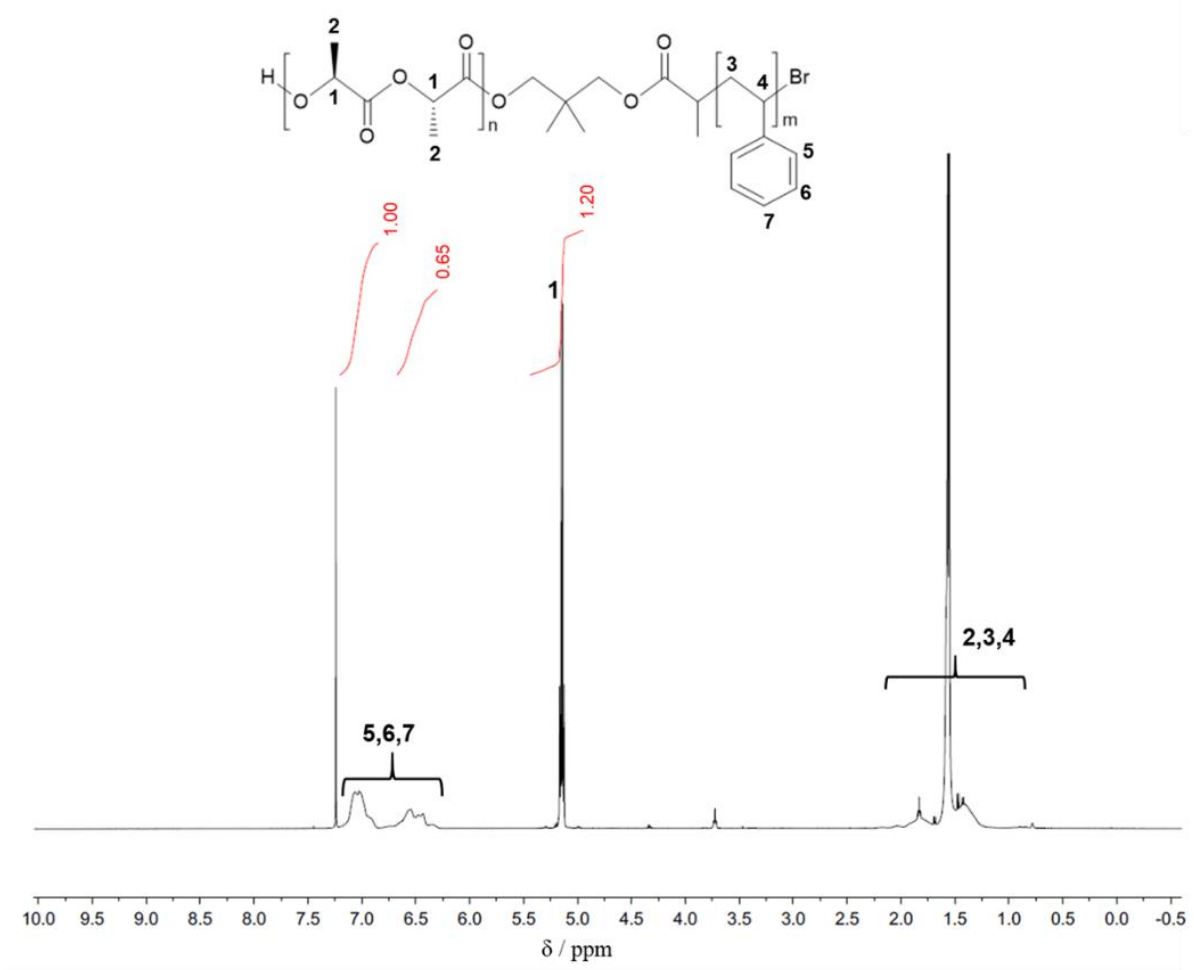

Figure S5. ${ }^{1} \mathrm{H}$ NMR spectrum (500 MHz) of PS10-PLLA24. The measurement was carried out using deuterated chloroform as a solvent at $25^{\circ} \mathrm{C}$.

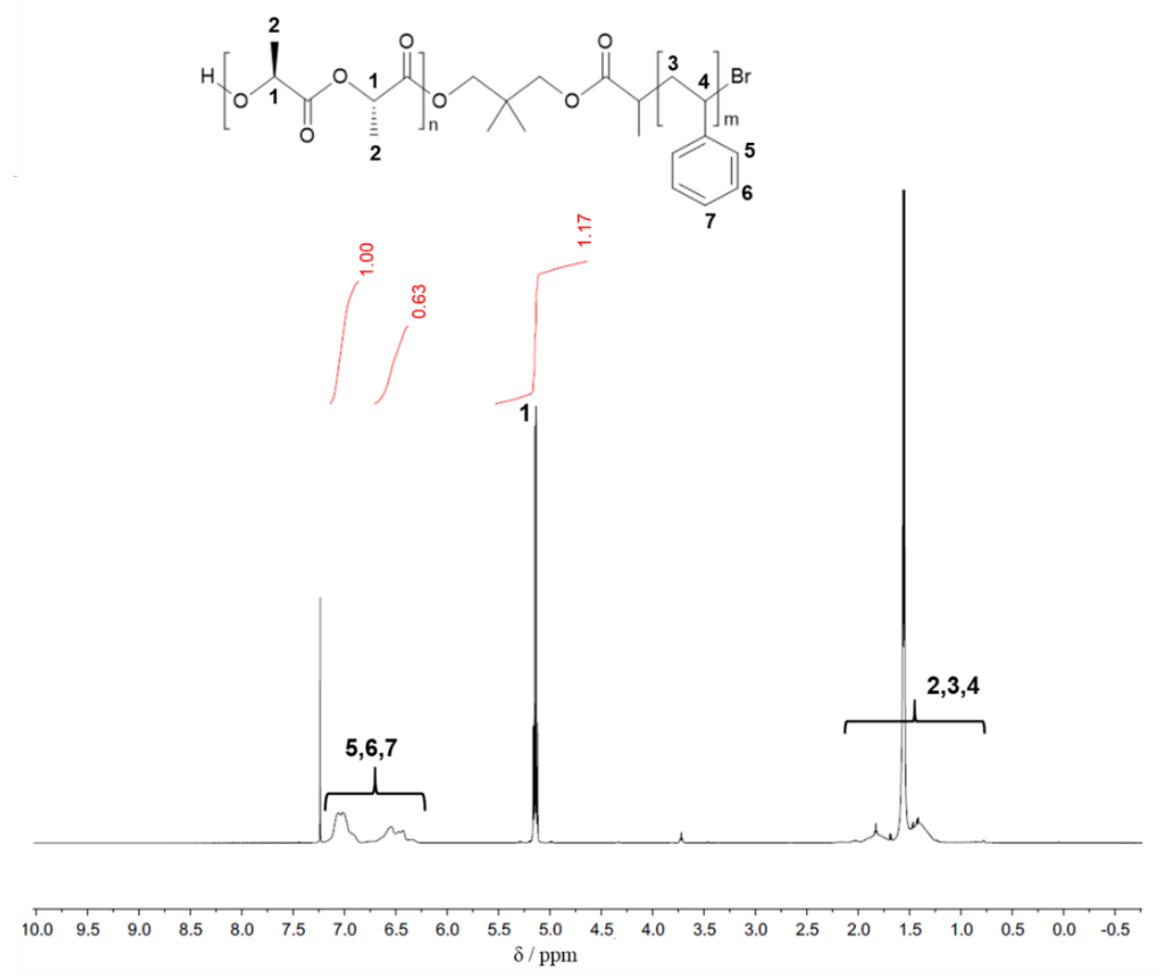

Figure S6. ${ }^{1} \mathrm{H}$ NMR spectrum $(500 \mathrm{MHz})$ of PS17-PLLA42. The measurement was carried out using deuterated chloroform as a solvent at $25^{\circ} \mathrm{C}$. 


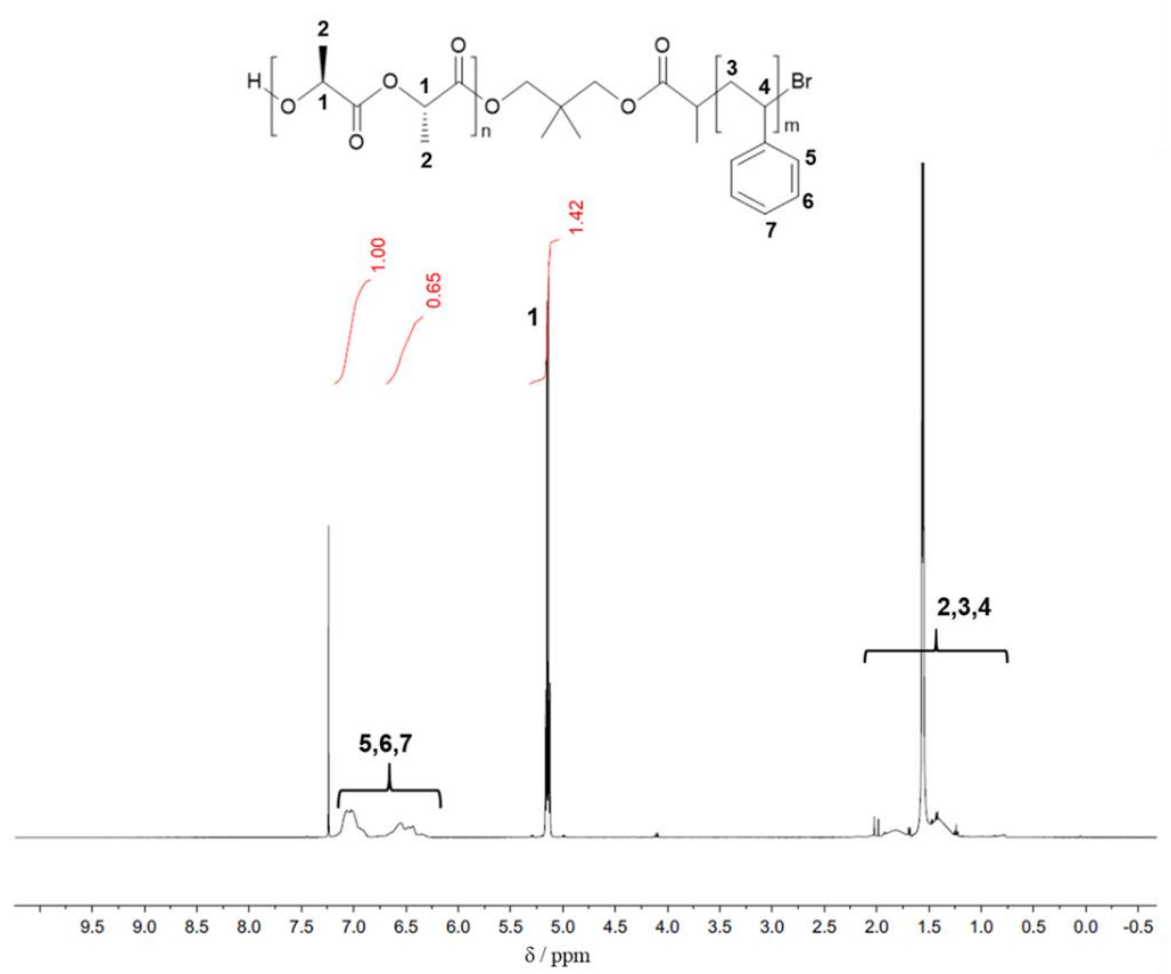

Figure S7. ${ }^{1} \mathrm{H}$ NMR spectrum (500 MHz) of PS24-PLLA67.The measurement was carried out using deuterated chloroform as a solvent at $25^{\circ} \mathrm{C}$.

Table S1. Characterization of synthesized PLLA-rich PS-PLLA samples with various molecular weights.

\begin{tabular}{|c|c|c|c|c|c|c|}
\hline \multirow{2}{*}{ Sample } & \multirow{2}{*}{$\begin{array}{c}\mathrm{M}_{\mathrm{n}}^{\mathrm{a}} \\
\left(\mathrm{g} \mathrm{mol}^{-1}\right)\end{array}$} & \multirow{2}{*}{$\bigoplus^{\mathrm{b}}$} & \multicolumn{2}{|c|}{$f^{v \mathrm{a}}$} & \multicolumn{2}{|c|}{$\mathrm{M}_{\mathrm{n}}^{\mathrm{a}}\left(\mathrm{g} \mathrm{mol}^{-1}\right)$} \\
\hline & & & PS & PLLA & PS & PLLA \\
\hline PS10-PLLA24 & 34,100 & 1.28 & 0.33 & 0.67 & 10,000 & 24,100 \\
\hline PS17-PLLA42 & 58,700 & 1.31 & 0.33 & 0.67 & 17,200 & 41,500 \\
\hline PS24-PLLA67 & 91,000 & 1.30 & 0.30 & 0.70 & 24,100 & 66,900 \\
\hline
\end{tabular}

${ }^{\text {a }}$ determined by ${ }^{1} \mathrm{H}$ NMR based on, $\rho_{P S}=1.05 \mathrm{~g} \mathrm{~cm}^{-3}, \rho_{\text {PLLA }}=1.25 \mathrm{~g} \mathrm{~cm}^{-3}$.

${ }^{\mathrm{b}}$ determined by GPC. 


\section{Thermal Properties}

To examine the thermal properties of the PLLA-rich PS-PLLA BCPs*, the bulk samples were examined by thermogravimetry (TGA) and differential scanning calorimetry (DSC). TGA thermograms with a plot of weight loss against temperature were carried out for PS10PLLA24, PS17-PLLA42, and PS24-PLLA67. As shown in Figure S8, two degradation steps can be identified at which the first weight loss starts approximately at $255^{\circ} \mathrm{C}$ for resulting from the weight loss of PLLA block. The thermogram appears a significant second drop, indicating that the degradation of PS approximately at $400{ }^{\circ} \mathrm{C}$. According to the results, the volume fraction of polylactides block for PS10-PLLA24, PS17-PLLA42, and PS24-PLLA67 are estimated to be $0.69,0.70$, and 0.71 , respectively, in line with the NMR results. The DSC measurements of the PS10-PLLA24, PS17-PLLA42, and PS24-PLLA67 show the Tgs of PLLA at approximately $56.7^{\circ} \mathrm{C}, 58.4^{\circ} \mathrm{C}$, and $59.6^{\circ} \mathrm{C}$, respectively (Figure S9) while the Tgs of PS blocks are overlapped by the exothermic peaks from crystallization of PLLA blocks, indicating the absence of confinement effect from PS on crystallization. Nevertheless, melting peaks can be found around the temperature ranging between $140{ }^{\circ} \mathrm{C}$ to $180{ }^{\circ} \mathrm{C}$; therefore, the crystallization of PLLA can be annihilated by thermal treatment as mentioned above.
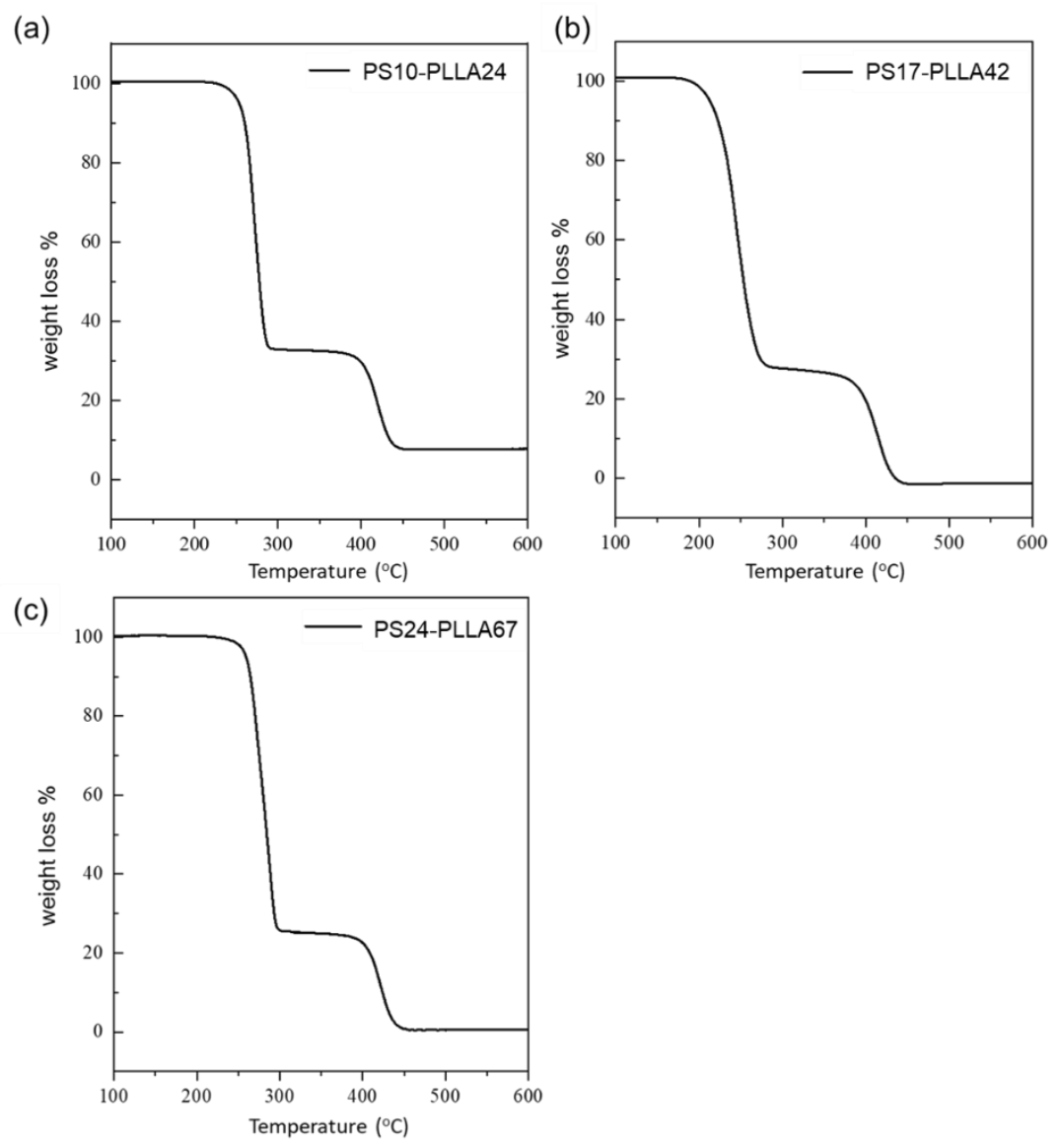

Figure S8. TGA thermograms of (a) PS10-PLLA24, (b) PS17-PLLA42 and (c) PS24-PLLA67. The heating rate is $10^{\circ} \mathrm{C} \mathrm{min}-1$. 


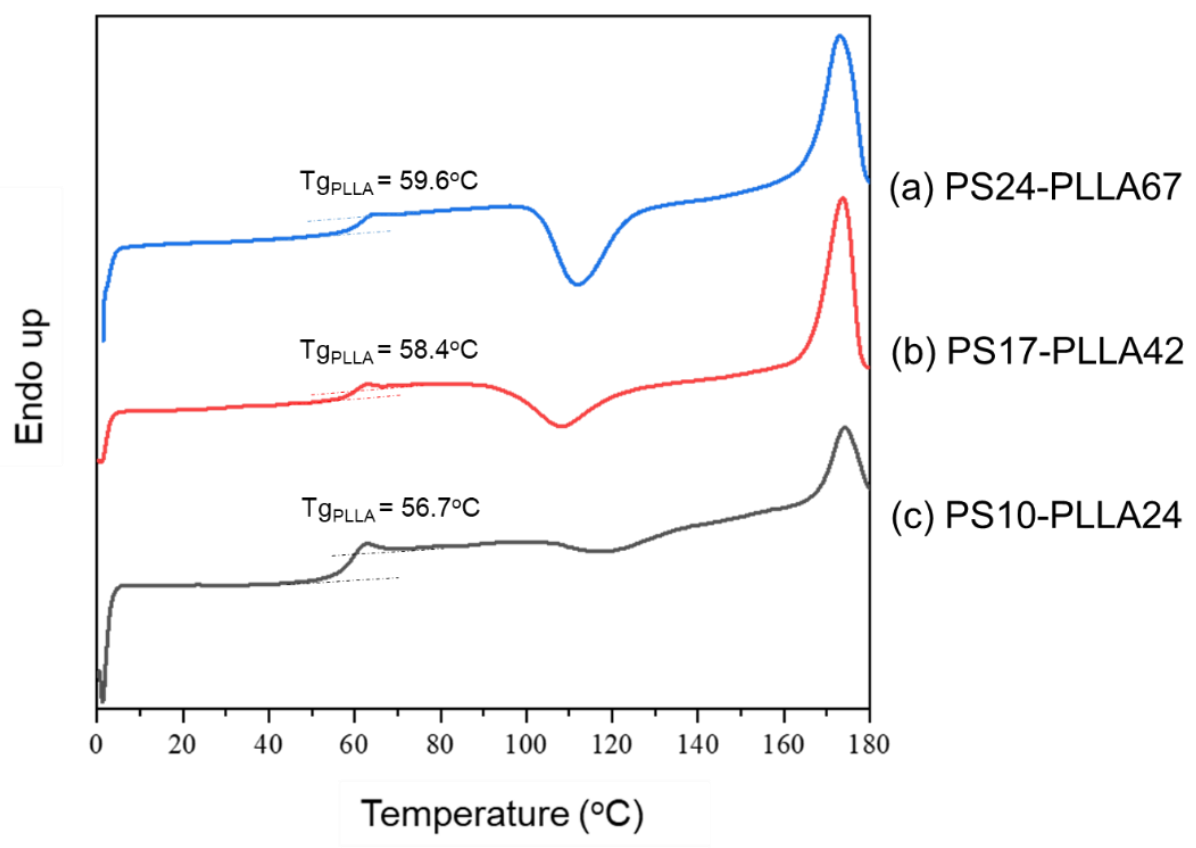

Figure S9. DSC thermograms of (a) PS10-PLLA24, (b) PS17-PLLA42 and (c) PS24-PLLA67 samples. The heating rate is $10{ }^{\circ} \mathrm{C} \mathrm{min}^{-1}$. 
3. Low magnification TEM image for curved multilayered lamellae.

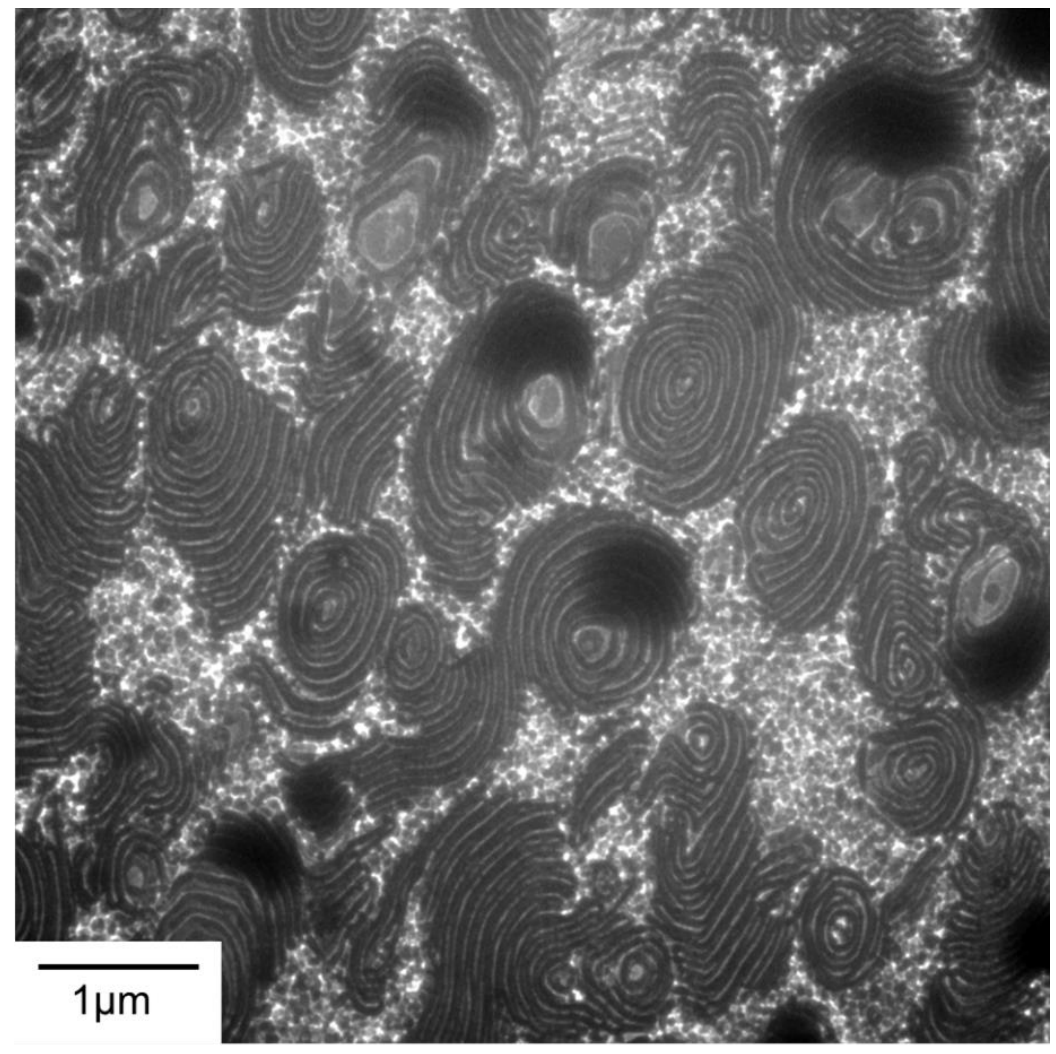

Figure S10. Low magnification TEM micrograph of self-assembled PS24-PLLA67 from solution casting with solvent evaporation rate of $0.04 \mathrm{ml} \mathrm{hr}^{-1}$ giving curved multilayered lamellae wiht both concentric and roll-cake texture. 


\section{Examination of crystallization effects on self-assembled hierarchical superstructures}

Wide-angle X-ray diffraction (WAXD) experiments of the solution-cast PLLA-rich PSPLLA after the thermal treatment as described above were carried out by Bruker D8A25 Phaser diffractometer using $\mathrm{Cu} \mathrm{K \alpha}$ radiation $\left(\lambda=0.154 \mathrm{~nm}\right.$ ) with $2 \theta$ angle scanning ranging from $10^{\circ}$ to $30^{\circ}$ at a step scanning of $0.2^{\circ} \mathrm{min}^{-1}$. As shown Figure S11a, a typical amorphous profile for the PLLA can be clearly identified, suggesting that the crystallization effect of PLLA on the self-assembled PS24-PLLA67 after thermal treatment should be insignificant. Similar results can be found in the solution-cast samples with lower solvent evaporation rates (Figures S11b and S11c).

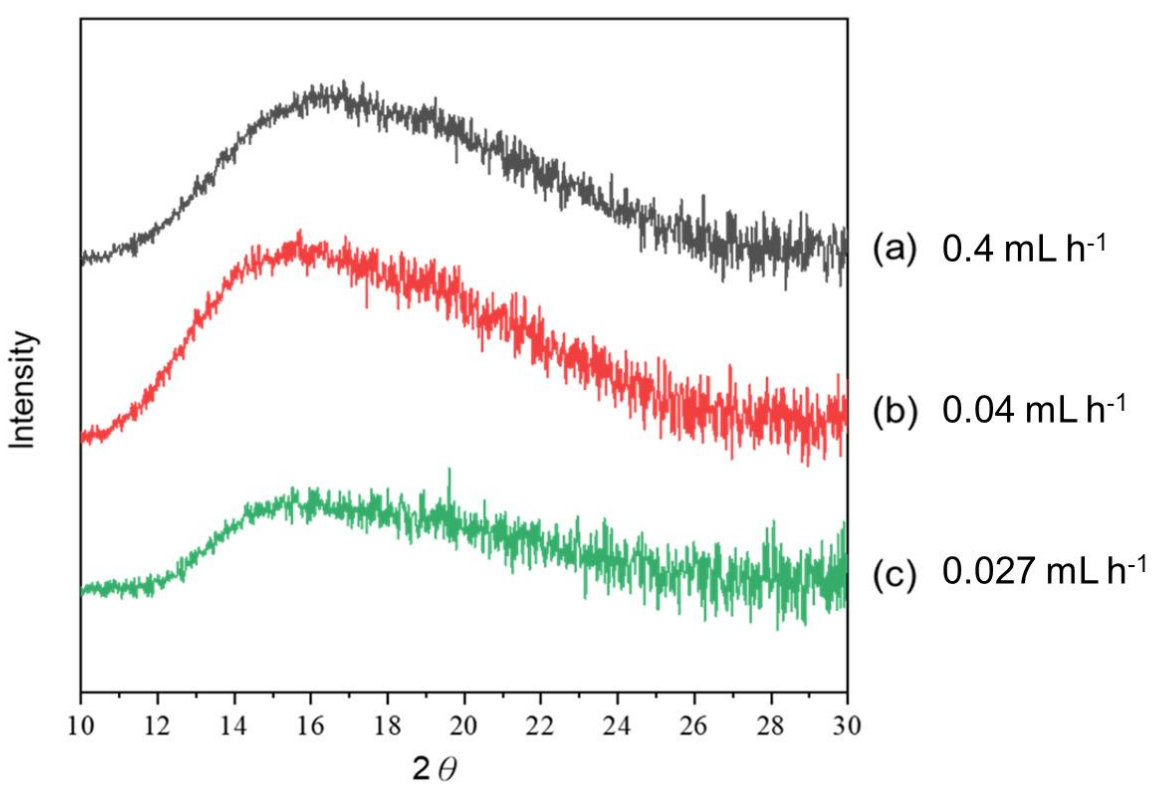

Figure S11. 1D WAXD profiles of solution-cast PS24-PLLA67 $\left(f_{\mathrm{PLLA}}{ }^{v}=0.70\right)$ after thermal annealing at $180{ }^{\circ} \mathrm{C}$ followed by rapid cooling at $150{ }^{\circ} \mathrm{C} \mathrm{min}^{-1}$ to $0{ }^{\circ} \mathrm{C}$. The solution-cast samples were prepared by solution casting with solvent evaporation rate of (a) $0.4 \mathrm{~mL} \mathrm{~h}^{-1}$; (b) $0.04 \mathrm{~mL} \mathrm{~h}^{-1}$; (c) $0.027 \mathrm{~mL} \mathrm{~h}^{-1}$. 


\section{Templated sol-gel reaction of multilayered lamellae structure}

Templated sol-gel reaction was carried out for the preservation of the PLLA microdomain(s) in the self-assembled PS-PLLA. By taking advantage of degradable character of the polylactide, nanoporous PS can be prepared after hydrolysis of PLLA in the PS-PLLA, and then used as a template for templated sol-gel reaction of tetraethyl orthosilicate (TEOS). The PS template was first immersed into the solution (sols) of the mixture of $\mathrm{TEOS} / \mathrm{HCl}_{(a q)}$ $(1 \mathrm{M}) /$ methanol (weight ratio $=10 / 1 / 25$ ) for one day at which the TEOS, $\mathrm{HCl}_{(a q)}$ and methanol were used as sol-gel reaction precursor, catalyst and solvent, respectively, for the replication of the PLLA microdomains by $\mathrm{SiO}_{2}$. Followed by the gelation with control humidity at $50{ }^{\circ} \mathrm{C}$ for 24 hours, the replicated morphologies could be directly visualized by scanning electron microscopy. As shown in Figure S12, after removal of the PS by UV exposure, curved lamellar textures can be clearly identified. There are two types of the curved lamellar textures; one appears as concentric columnar-like morphology (Figure S12a) and the other gives spiral-like morphology (Figure S12b). Those morphological results are in line with the suggested curved multilayered lamellae with concentric lamellar and roll-cake textures.
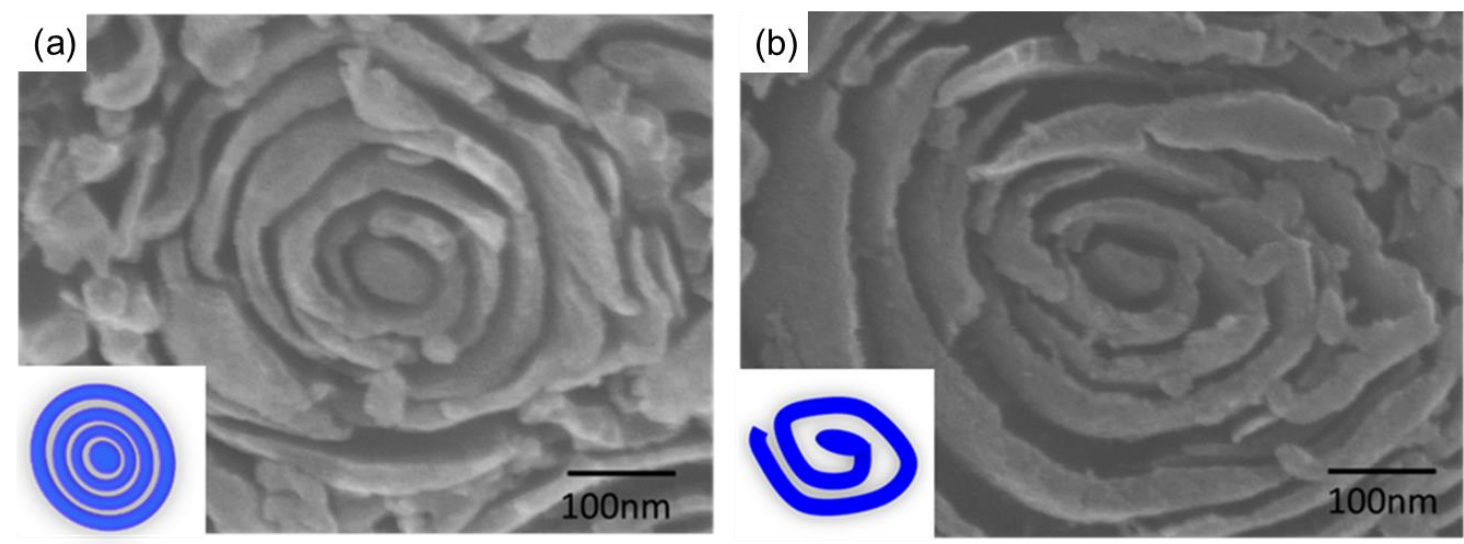

Figure S12. SEM micrographs of nanostructured $\mathrm{SiO}_{2}$ fabricated by templated sol-gel reaction using nanoporous PS as a template prepared by hydrolysis of self-assembled PS24-PLLA67 with concentric lamellar texture (a) and roll-cake texture (b), followed by removal of PS through UV exposure. The insets show the corresponding illustrations of the forming textures. 


\section{Morphological evolution of PLLA-rich PS-PLLA from self-assembly}
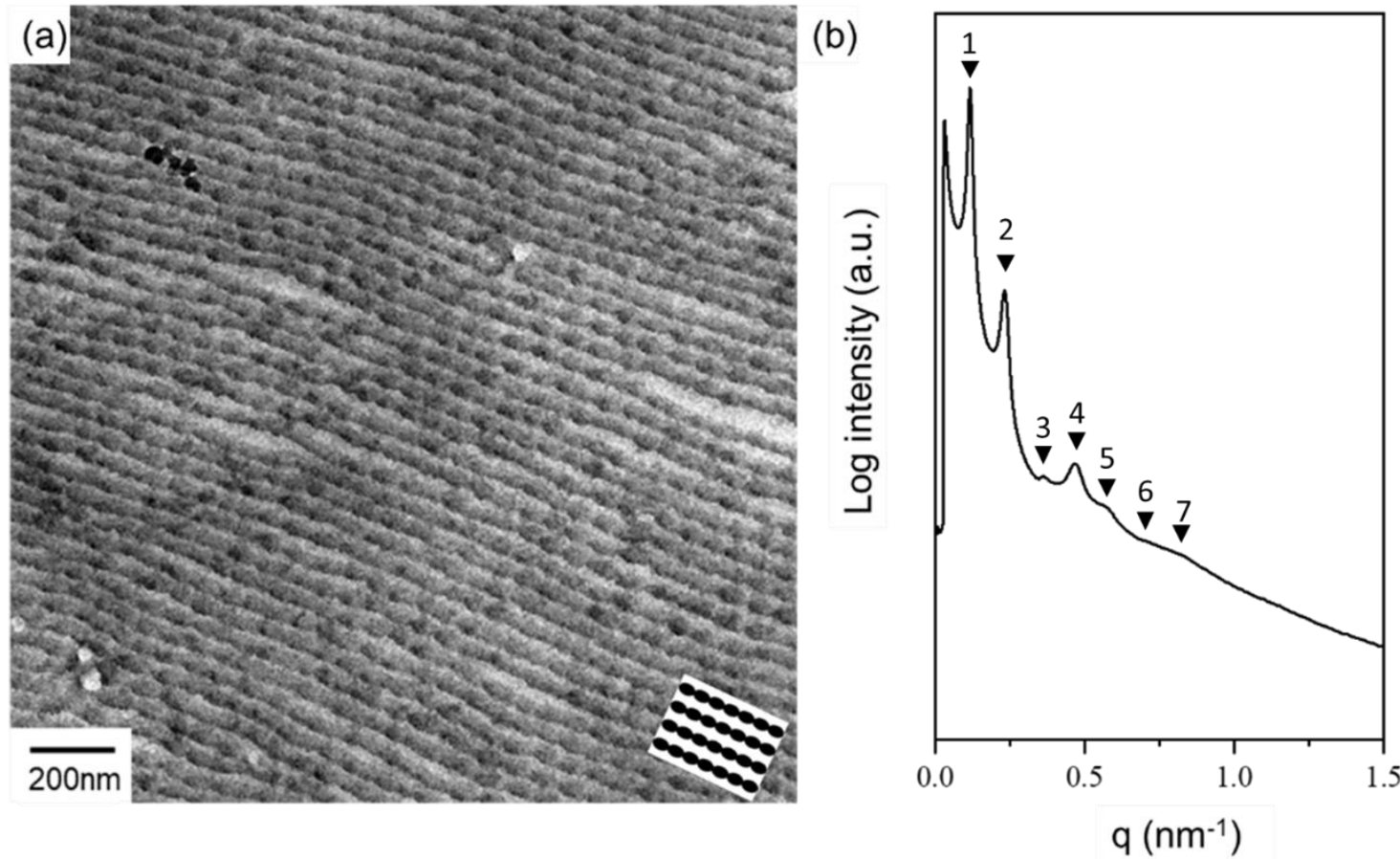

Figure S13. (a)TEM micrograph and (b) SAXS result of self-assembled PS24-PLLA67 from solution casting with extremely slow solvent evaporation rate at $0.0008 \mathrm{ml} \mathrm{hr}^{-1}$. Undulated lamellae with long-range order can be observed by TEM along with the appearance of reflections at high $q$ region at which the $d$-spacing was approximately $54 \mathrm{~nm}$ determined by the primary reflection in the SAXS profile. The inset shows the corresponding illustration of the forming texture. 


\section{PLLA-rich PS-PLLA samples with equivalent volume fraction but different molecular weights}

To generalize the self-assembling behaviors of the BCPs*, in comparison with the ones with PS-rich PS-PLLA composed of an achiral polystyrene (PS) and a chiral poly(L-lactide) (PLLA) as major and minor blocks, respectively, PLLA-rich PS-PLLAs with equivalent volume fraction but different molecular weights (PS10-PLLA24 and PS17-PLLA42 beside PS24-PLLA67) as listed in Table S1 were synthesized. Similar to the PS24-PLLA67, the TEM projection results of the PS10-PLLA24 and PS17-PLLA42 (Figure S14) suggest the formation of curved multilayered lamellae after solution casting followed with thermal annealing at 180 ${ }^{\circ} \mathrm{C}$ and rapid cooling at $150{ }^{\circ} \mathrm{C} \mathrm{min}^{-1}$ to $0{ }^{\circ} \mathrm{C}$. The lamellar textures were further confirmed by SAXS results (Figure S15) at which the relative $q$ values of 1:2:3:4 can be identified in both samples. The imaging and scattering results suggest the generic formation of curved multilayered lamellar phase in the PLLA-rich PS-PLLA. In contrast to the $d$-spacing of PS24PLLA67 (45.9 nm), PS10-PLLA24 and PS17-PLLA42 give the spacings of $34.3 \mathrm{~nm}$ and 39.1 $\mathrm{nm}$, respectively.

(a)

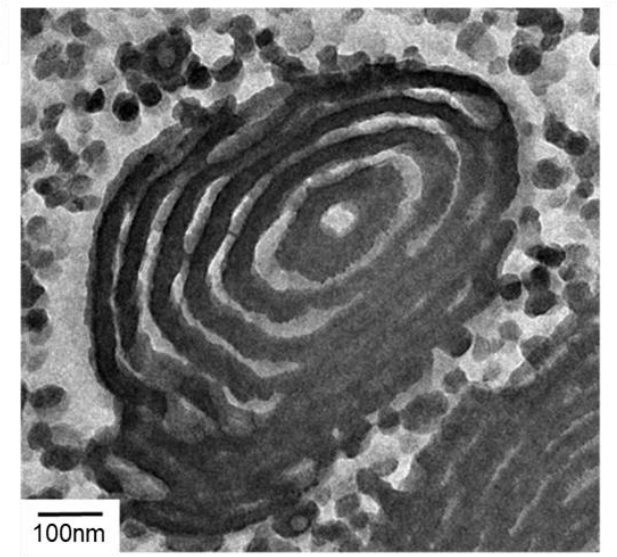

(b)

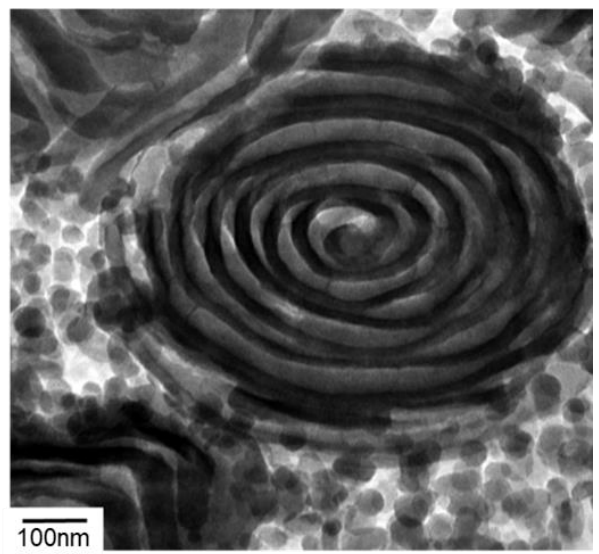

Figure S14. TEM micrographs of self-assembled (a) PS10-PLLA24 and (b) PS17-PLLA42 from solution casting after thermal annealing at $180{ }^{\circ} \mathrm{C}$ followed by rapid cooling at $150{ }^{\circ} \mathrm{C}$ $\min ^{-1}$ to $0{ }^{\circ} \mathrm{C}$. The PS microdomains appear dark and PLLA microdomains appear bright due to $\mathrm{RuO}_{4}$ staining. 


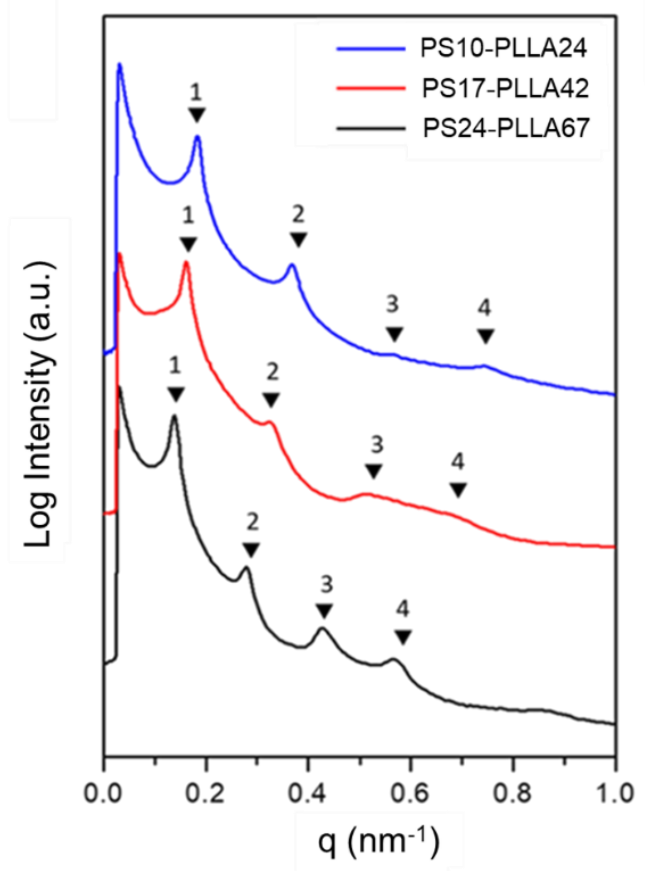

Figure S15. 1D SAXS profiles of self-assembled PS24-PLLA67, PS10-PLLA24 and PS17PLLA42 from solution casting after the thermal treatment with the same sample preparation procedure. 
8. Evidence for inter-chain chiral interactions of self-assembled PLLA-rich PS-PLLA
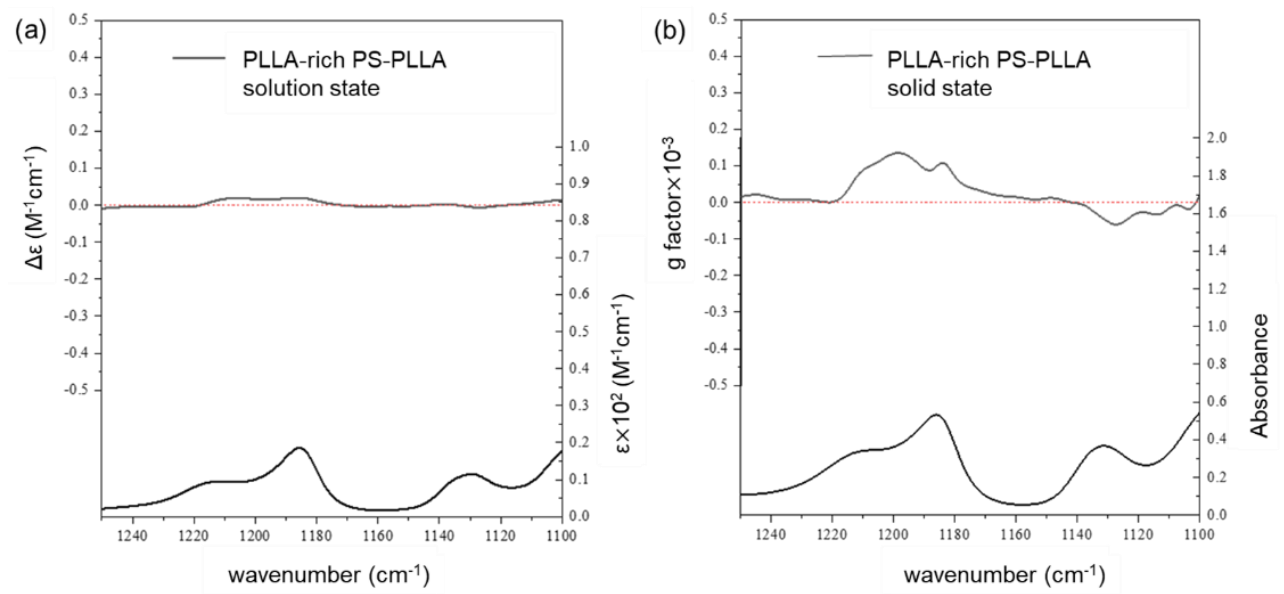

Figure S16. VCD and corresponding FTIR absorption spectra of $\mathrm{C}-\mathrm{O}-\mathrm{C}$ vibrational mode of PLLA-rich PS24-PLLA67 in the (a) DCM solution and (b) solid states. The concentration of solution is $2 \mathrm{wt} \%$. 


\section{Anisotropic effect on VCD spectrum}

There is no significant variation on the vibrational linear dichroism (VLD) signals from wavenumber $1250 \mathrm{~cm}^{-1}$ to $1100 \mathrm{~cm}^{-1}$ for PLLA-rich PS-PLLA (Figure S17), indicating that there is no significant anisotropic effect on spectroscopic measurements in the solution and the solid states. Those results assure that the VCD signals are mainly contributed by the chiral interactions from chiral blocks.
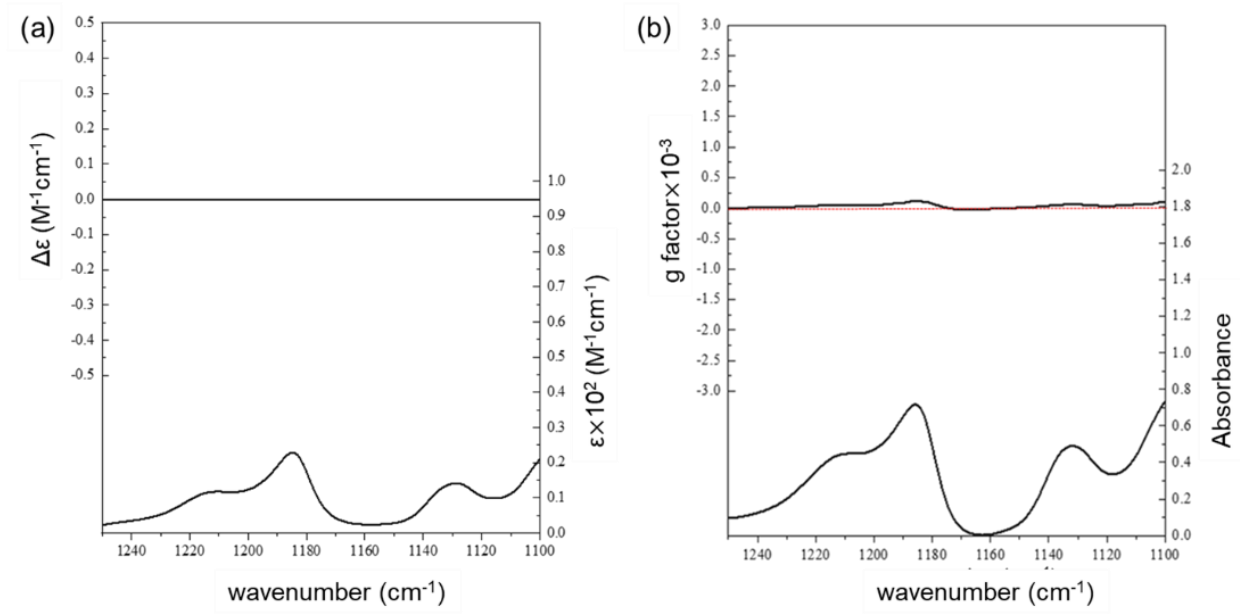

Figure S17. VLD and corresponding FTIR absorption spectra of C-O-C vibrational mode of PLLA-rich PS24-PLLA67 in the (a) DCM solution and (b) solid states. The concentration of solution is $2 \mathrm{wt} \%$. 